\title{
Mechanical properties and dislocation nucleation in nanocrystals with blunt edges
}

\author{
Jonathan Amodeo** and Khalid Lizoul ${ }^{12}$ \\ Université de Lyon, INSA-Lyon, MATEIS UMR5510 CNRS, 69621 Villeurbanne, France \\ ¿Université de Lyon, Université Jean Monnet, LASPI EA3059, 42023 Saint-Etienne, France
}

To be published in:

Materials and Design

(*) Corresponding author:

Dr. Jonathan Amodeo

INSA-Lyon, Laboratoire MATEIS

25 avenue Jean Capelle

Bat. St. Exupéry, $2^{\text {ine étage }}$

69621 Villeurbanne Cedex, France

Tel: 0472438235

Fax: 0472438539

e-Mail: jonathan.amodeo@insa-lyon.fr 


\title{
Mechanical properties and dislocation nucleation in nanocrystals with blunt edges
}

\begin{abstract}
The deformation of blunt nanocrystals under uniaxial compression is modelled using molecular dynamics simulations. $\mathrm{Ni}_{3} \mathrm{Al}$ blunt nanoparticles are built using a simple algorithm that allows continuous shape design from perfectly-sharp nanocubes to nanospheres including a large set of cuboid shapes. Simulation results show that blunt nanoparticles are significantly stronger in comparison to their perfectly cubic counterparts and deform due to the nucleation of partial dislocations and deformation twins. Furthermore, while the early plastic deformation of nano-objects is generally attributed to the nucleation of dislocations from the surface, here we show that homogeneous dislocation nucleation may also happen in blunt nanoparticles and a size-dependent transition parameter is defined. This study emphasizes how much the design of virtual samples is crucial when modelling nano-objects mechanical properties using molecular dynamics simulations and provides new insights onto potential incipient plasticity features at the nanoscale.
\end{abstract}

Keywords : nanoparticles, sample design, nanomechanics, molecular dynamics

\section{Introduction}

Among the different nano-objects as nanowires, nanopillars, nanoparticles (NPs) and thin films, NPs are one of those with the richest amount of functional capabilities. Indeed, they are used in various domains of application as nano electro-mechanical systems (NEMS) [1,2], biological detectors and sensors $[3,4]$. They make also good drug delivery systems and catalysts in the field of pharmaceutics $[5,6]$ as well as strong friction-reducer agents in lubricants $[7,8]$. In all these fields, the targeted functional property depends strongly on the NP shape and mechanical state. Within this framework, NPs and other nano-objects exhibit particularly promising mechanical properties: they often show a size-dependent elastic regime $[9,10]$ as well as increased yield strength and 
ductility compared to their bulk counterpart [11,12]. To improve the understanding of such small-scale mechanical features, several groups recently focus on the development of nanomechanical tests merely based on scanning and transmission electron microscopy (SEM and TEM) which are believed to be the more suited experimental techniques at such length scales $[13,14]$. In spite of their space and time scale constrains, molecular dynamics (MD) simulations are extensively used in parallel to these two experimental approaches to provide theoretical evidences of elementary plasticity mechanisms [15-18].

In Mordehai et al. [19], the authors investigate the mechanical properties of faceted gold NPs under compression using SEM, MD and finite-element compression simulations. MD simulation results emphasize the nucleation of $1 / 6<112>\{111\}$ Shockley partial dislocations from the vertices as the main plastic deformation mechanism and a significant size-effect is revealed. Later, Bian and Wang have studied the deformation of perfectly shaped copper nanospheres using a similar MD approach [20]. While the nucleation of partial dislocations is once again the main incipient plasticity process, the authors also show that it further leads to nanotwinning as already observed in other FCC nanostructures $[11,13,21]$. The mechanical properties of perfectly shaped $\mathrm{Ni}_{3} \mathrm{Al}$ nanocubes (NCs) have also been investigated using $\mathrm{MD}[22,23]$ and compared to $\mathrm{Ni}_{3} \mathrm{Al}-$ based $\gamma^{\prime}$ precipitates extracted from a CMSX-4 superalloy further compressed within the SEM [24,25]. Again, the main plasticity process occurring in the simulation is the heterogeneous nucleation from the surface (NCs edges and corners in this case) of partial dislocations that further lead to a pseudo-twin (PT) structure, typical of L1 2 crystals $[26,27]$. Overall, these studies show that sharply-designed and single crystalline NPs deform under ultra-high stress $(1<\sigma<20 \mathrm{GPa})$ thanks to the surface nucleation of dislocations and twins from the sample surface.

In this context, one can wonder whether virtual samples are designed with enough accuracy to quantitatively model surface-based processes and reproduce experimental mechanical conditions. Indeed and for the sake of convenience, samples designed for MD nanomechanical tests rarely provide such a high level of details: virtual NPs and nanowires generally exhibit flat facets bounded by sharp edges and vertices (see among other refs. 
$[19,22,28]$ ), whilst nanospheres are most of the time perfectly and symmetrically shaped (see e.g. refs. $[20,29,30])$, in contrast to several experimental evidences emphasizing more blunt and rounded shapes [31-33]. This question particularly applies at the nanoscale where surfaces and interfaces may act as sinks and sources for dislocation-driven plasticity processes due to stress concentration [34,35].

In this study, we confront the fundamental question of edge shape investigating the mechanical response and the elementary plasticity processes of blunt nanocrystals under compression using a large set of MD simulations. For this purpose, NPs with rounded edges and corners are designed using a simple algorithm based on a blunting coefficient $\alpha$ as detailed in the method section. While we will show that the main results are not expected to depend on the crystalline structure, we consider here $\mathrm{L}_{2} \mathrm{Ni}_{3} \mathrm{Al} \mathrm{NCs}$.

Besides $\mathrm{Ni}_{3} \mathrm{Al}$-based blunt $\mathrm{NCs}$ are the main strengthening component of $\gamma / \gamma^{\prime}$ nickel based superalloys, the $\mathrm{L1}_{2}$ structure appears as the ideal candidate to quantify the influence of shape blunting on the incipient plasticity mechanisms as it features a wider panel of dislocations than usual crystalline structures. $\mathrm{Ni}_{3} \mathrm{Al}$ plasticity is mainly due to $<110>$ Burgers vector superdislocations that possibly dissociate in: (i) four $1 / 6<112>\{111\}$ partial dislocations separated by two complex stacking faults (CSF) bounding an anti-phase boundary (APB), (ii) five $1 / 6<112>\{111\}$ partial dislocations involving two CSFs, two APBs and one super intrinsic stacking fault (SISF), (iii) two $1 / 3<112>$ dislocations that circumscribe an SISF. $<112>$ dislocations may also dissociate in four $1 / 3<112>$ dislocations separated by SISF and super extrinsic stacking fault (SESF) in the $\{111\}$ planes. In addition, <110> superdislocations can also glide in cubic $\{100\}$ plans (see refs. [36,37] for a more exhaustive list of possible dislocation dissociation processes) and $\{111\}$ pseudo-twinning (PT) as well as regular twinning have also been observed [26].

Here we will show that blunt NPs are significantly stronger than their sharp counterpart and that a transition from heterogeneous-to-homogeneous dislocation nucleation happen under certain conditions of shape and size. This theoretical study provides new insights on the plasticity mechanisms and mechanical strength of nanoobjects. 


\section{Simulation methods}

In this paper, we perform MD simulations to investigate the deformation of initially dislocation-free blunt $\mathrm{Ni}_{3} \mathrm{Al}$ NPs under compression using the open-source code LAMMPS [38]. Most of the calculations are carried out using the Du, Wang and Yu EAM interatomic potential (referred as DWY in the text) [39] and some of them are also performed using the Angelo, Moody and Baskes (AMB) parameterization [40,41]. Both are known to reproduce $\mathrm{Ni}_{3} \mathrm{Al}$ elastic properties and defect energies with good accuracy and are especially suited for nanocompression simulations [23]. For more details, $\mathrm{Ni}_{3} \mathrm{Al}$ elastic properties, stacking fault and surface energies computed with the DWY and AMB parameterizations as well as with two additional interatomic potentials $[42,43]$ are provided as supplementary information (see Table S1).

All samples are $<100>$-oriented NPs carved from a $\mathrm{Ni}_{3} \mathrm{Al}$ block of atoms with the $\mathrm{L}_{2}$ crystalline structure.

$\mathrm{Ni}_{3} \mathrm{Al} \mathrm{NPs}$ are all $\{100\}-\mathrm{Ni}_{0} \mathrm{Al}_{0}$ terminated as it has the lower surface energy compared to $\{100\}-\mathrm{Ni}$ terminated surfaces. In order to investigate the role of the sample shape, NCs corners and edges are rounded following a two-steps process. First, virtual spheres of radius $R$ are included flush with each corner of a perfectly shaped $\mathrm{NC}$ and exceeding atoms are cut out (Figure 1). Then, a similar approach is used to further round sample edges using virtual cylinders of same radius. A normalized blunting parameter $\alpha=\frac{2 R}{L}$ (where $L$ is the original NC edge length) is defined. As shown Figure 1, starting from an originally sharp NC $(\alpha=0)$, increasing $\alpha$ leads to blunt NPs $(0<\alpha<1)$ made of gradually rounded corners and edges, up to a perfect nanosphere for $\alpha=1$. This study covers NP sizes from $L=5$ up to $35 \mathrm{~nm}$, with $\alpha$ values ranging from 0 to 1 with a particular focus on the 0 to 0.5 range.

Preliminary to the compression test, the NP energy is minimized down to a force norm of $10^{\mathrm{s}} \mathrm{eV} / \AA$. The sample is thus equilibrated during 30 ps using standard MD in the NVE ensemble plus 50 ps NVT using the NoséHoover thermostat. A time step of $2 \mathrm{fs}$ is used for all MD runs and free boundary conditions are applied. 
Then, compression is performed using two infinite top and bottom force fields which behave respectively as flat punch indenter and substrate (see refs. [22,44] for more details). The top indenter is displaced at a constant rate equivalent to an engineering strain rate of $10^{\S} \mathrm{S}^{-1}$ for all particle sizes, while the bottom indenter is kept fixed. Compression simulations are carried out at $5 \mathrm{~K}$ and $300 \mathrm{~K}$ constant temperatures without quantitative changes in terms of yield stress and deformation processes (see supplementary Figures S1 and S2 for more details). The force $f$ acting on the top indenter is recorded on-the-fly during compression. The (engineering) compressive stress $\sigma$ is defined as the $f / S_{o}$ ratio where $S_{o}$ is the initial top surface area made of first layer atoms (see Fig. 1) that is computed using a convex Hull approach [45]. While the use of the compressive stress is somehow standard in nano- and micro-compression tests, we will discuss later how significant is the stress definition at such length-scales. 
a)

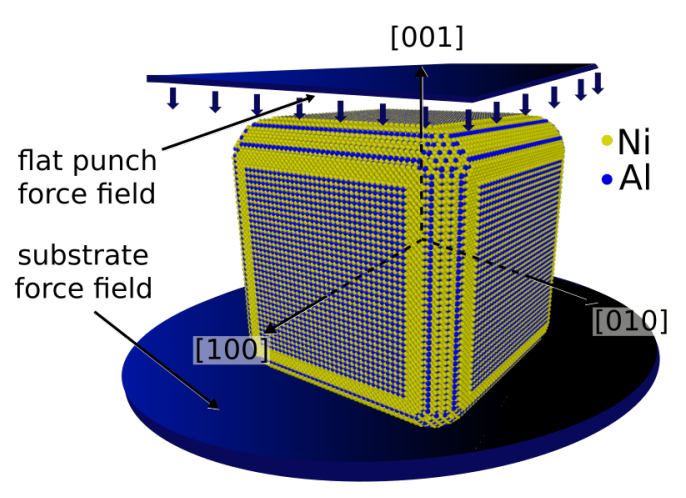

b)

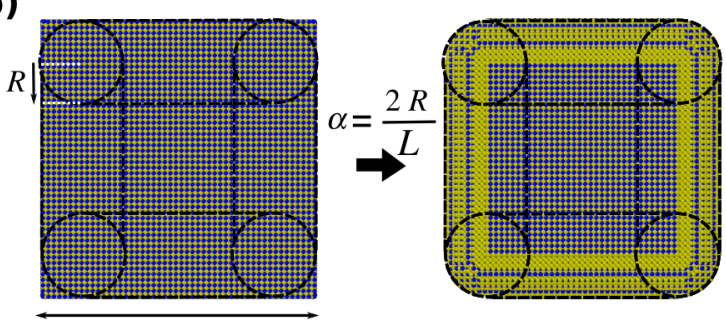

L

c)
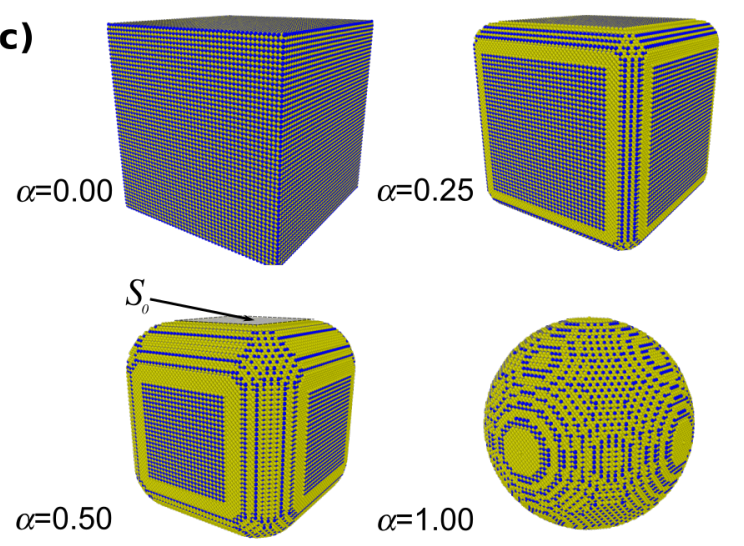

Fig. 1 (color online) NP compression simulation protocol and blunting method. a) The simulation setup is made of a single-crystalline NP surrounded by two force fields that behave as indenter (flat punch) and substrate respectively, b) The blunting method consists in including virtual spheres and cylinders inside a perfectly shaped NC and remove all exceeding atoms. $R$ is the cutting radius and $L$ the original NC edge length, $c$ ) Examples of NPs for few values of $\alpha . S_{0}$ describes the top surface area used to define the compressive stress.

Local "atomic" stresses are computed using the LAMMPS stress-per-atom compute for which the elementary volume associated to each atom is defined using a Voronoï tessellation approach. The (engineering) strain $\varepsilon$ is defined as the relative variation of the NP maximum size along the [001] direction. The analysis of lattice 
defects in $\mathrm{Ni}_{3} \mathrm{Al}$ is performed using a dedicated modified bond-angle distribution (BAD) analysis as described in ref. [22]. Nye tensor and skeletonization algorithms are used for dislocation identification [46,47]. Atomic configurations are visualized using AtomViewer [48] and Ovito [49] softwares.

\section{Results}

\subsection{Mechanical response of blunt NPs}

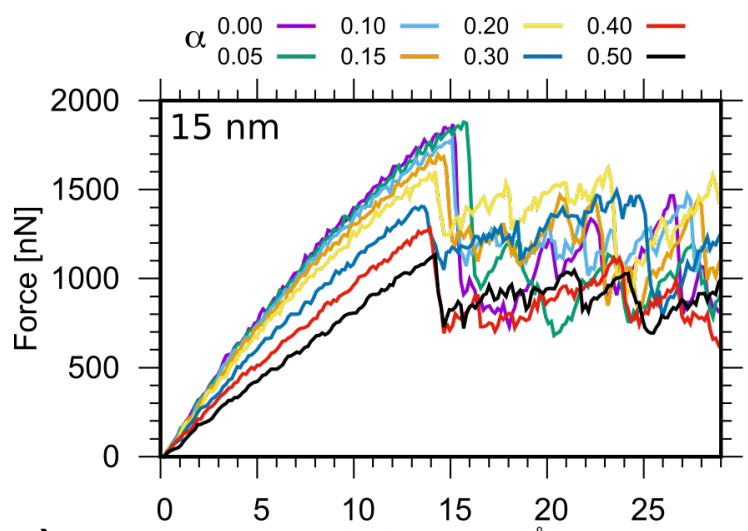

a)

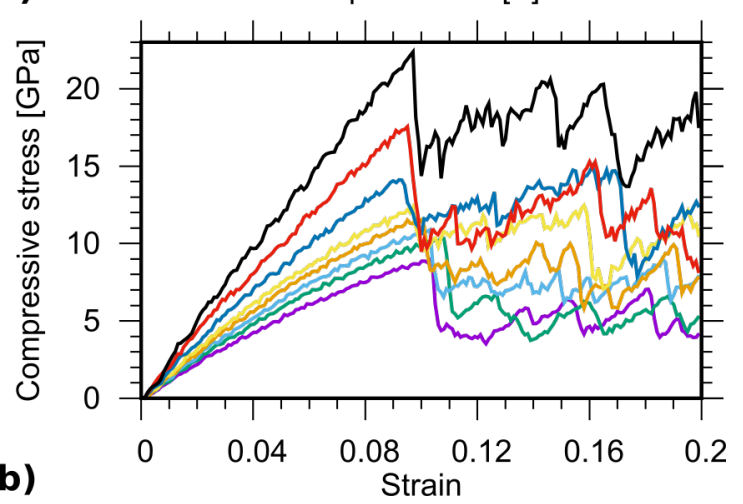

Fig. 2 (color online) Mechanical response of 15nm Ni Al NPs under compression. a) Force - displacement and b) stress - strain curves using the DWY parameterization. The blunting coefficient a ranges from 0 to 0.5 .

Figure 2 shows force - displacement and stress - strain curves for $L=15 \mathrm{~nm}$ NPs characterized by $\alpha$ values ranging from 0 (perfect NC) up to 0.5 (blunt NP). All the curves exhibit the same two deformation regimes i.e. an initial slightly non-linear elastic stage up to $\sim 10 \%$ strain $(\sim 15 \AA$ depth $)$ followed by a drop that underlines the beginning of a serrated plastic regime. On Figure $2 \mathrm{a}$ illustrating force - displacement curves, the 
yield force decreases with increasing $\alpha$, while the stress - strain curves (Fig. 2b) exhibit the opposite behaviour due to a faster diminution of the contact surface area compared to the induced force response. For $15 \mathrm{~nm}$ stress - strain curves, the critical yield stress $Y^{*}$ ranges from $9.0 \mathrm{GPa}$ up to to $22.4 \mathrm{GPa}$ with increasing $\alpha$ up to 0.5 and the Young's modulus $E$ varies from 115.6 up to $259.4 \mathrm{GPa}$ around the bulk value $E_{\text {buu }}=128.8 \mathrm{GPa}$ ( $E_{\text {but }}$ is computed using DWY elastic constants and the anisotropic elastic theory). These results confirm that shape matters: rounding edges and corners makes the NPs stiffer and stronger. The computed values of $Y$, yield force $f$ and $E$ for the $L=15 \mathrm{~nm}$ NPs are gathered Table 1 .

\begin{tabular}{|c|c|c|c|}
\hline$\alpha$ & $f^{e}[\mathrm{nN}]$ & $Y^{*}[\mathrm{GPa}]$ & $E[\mathrm{GPa}]$ \\
\hline 0.00 & 1866 & 9.0 & 115.6 \\
\hline 0.05 & 1878 & 10.4 & 129.7 \\
\hline 0.10 & 1784 & 10.9 & 141.6 \\
\hline 0.15 & 1685 & 11.6 & 153.7 \\
\hline 0.20 & 1602 & 12.3 & 162.9 \\
\hline 0.30 & 1404 & 14.1 & 185.9 \\
\hline 0.40 & 1282 & 17.5 & 222.1 \\
\hline 0.50 & 1134 & 22.4 & 259.4 \\
\hline
\end{tabular}

Additional stress - strain curves for NPs of 5, 10, 17.8 and 29.6 nm sizes are shown Figure 3.

No size-effect for perfectly sharp NCs is noticed in the investigated range of sizes what confirms previous observations for $\mathrm{Ni}_{3} \mathrm{Al} \mathrm{NCs}[22,23]$. Higher values of $\alpha$ show only slight variations of $Y^{*}$ with changing $L$ except for the $29.6 \mathrm{~nm}$ NPs which show strength softening for $\alpha=0.3$ to 0.5 . These variations vanish when 
comparing simulations performed at constant blunting radius $R$ what confirms that the surface shape is the main strength limiting factor of blunt NPs.

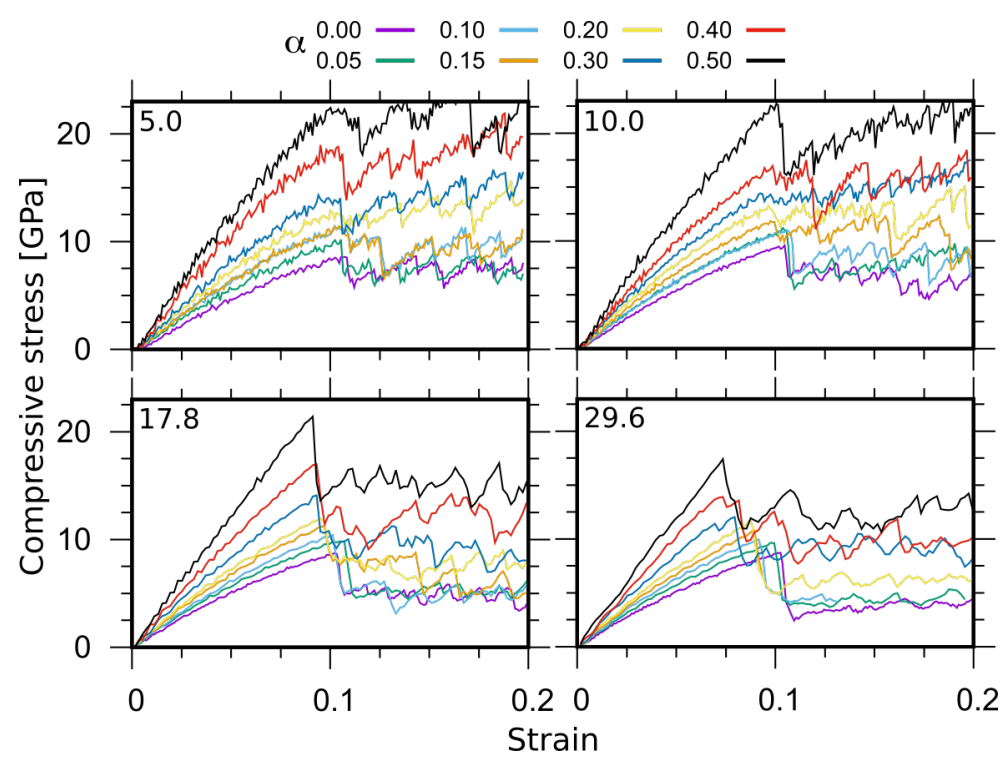

Fig. 3 (color online) Stress - strain curves for L=5, 10, 17.8 and $29.6 \mathrm{~nm}$ blunt NPs under compression using the DWY parameterization. The blunting coefficient a ranges from 0 to 0.5 .

\subsection{Plastic deformation mechanisms for low- $\alpha$ values}

The plastic deformation process related to the first stress drop of perfectly cubic $\mathrm{Ni}_{3} \mathrm{Al}$ NPs $(\alpha=0)$ corresponds to the heterogeneous nucleation from the surface (e.g. from a corner or an edge) of $1 / 6<112>\{111\}$ partial dislocations (Fig. 4). Due to the [001] compression conditions, partial dislocations nucleate in the four $1 / 6<112>\{111\}$ slip systems with the higher Schmid factor $M \sim 0.47: 1 / 6[1 \overline{1} 2](1 \overline{1} \overline{1}), 1 / 6[\overline{1} \overline{1} 2](111)$,

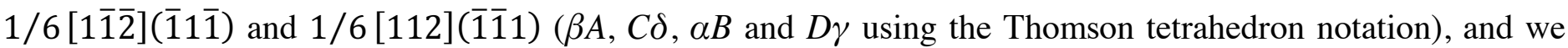
confirm here that NCs corners are the preferential nucleation sites for the two DWY and AMB potential parameterizations as shown Fig. 4a,b. After their nucleation, partial dislocations can either reach the opposite facets or interact with dislocations or stacking faults from other $1 / 6<112>\{111\}$ slip systems what leads in both cases to the storage of extended CSFs inside the sample. Afterwards, parts of the CSFs lead to PT (Fig. 4c) due to the subsequent nucleation of twinning partial dislocations gliding in adjacent slip plans. While the PT 
growing process accommodates the plastic deformation, it also acts as barrier for the propagation of partial dislocations gliding in secant slip systems as shown Fig. 4c. Please note that the partial dislocations responsible for the PT growth are nucleated in the same slip system than the former CSF.

Surface dislocation nucleation also apply in blunt NPs except that defects do not nucleate exactly at the corner anymore, but close to it, beneath the contact surface (Fig. 4d, see inset) due to the blunt shapes of edges and corners. At larger strains, CSFs are not only PT precursors anymore as some of them also lead to APBs due to the uncorrelated nucleation and propagation of complementary $1 / 6<112>\{111\}$ partial dislocations within the same glide plane (see e.g. Fig. 4e). The $\mathrm{L1}_{2}{ }^{-} \mathrm{CSF}-\mathrm{APB}-\mathrm{CSF}-\mathrm{L} 1_{2}$ stacking sequence which is typical of the $\mathrm{L} 1_{2}$ Burgers vector $\vec{b}=<110>$ decomposition [36] is observed. On the other hand, very few SISFs occur. 


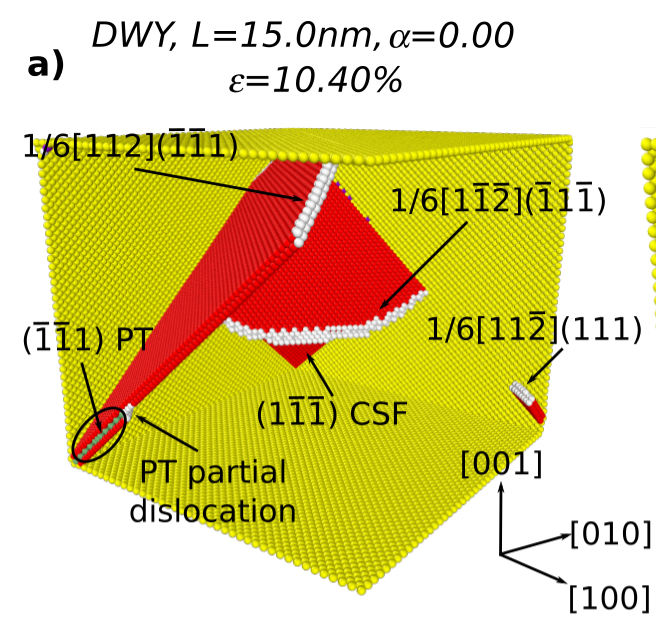

b) $\underset{\varepsilon=5.47 \%}{A M B, L=10.0 \mathrm{~nm}, \alpha=0.00}$
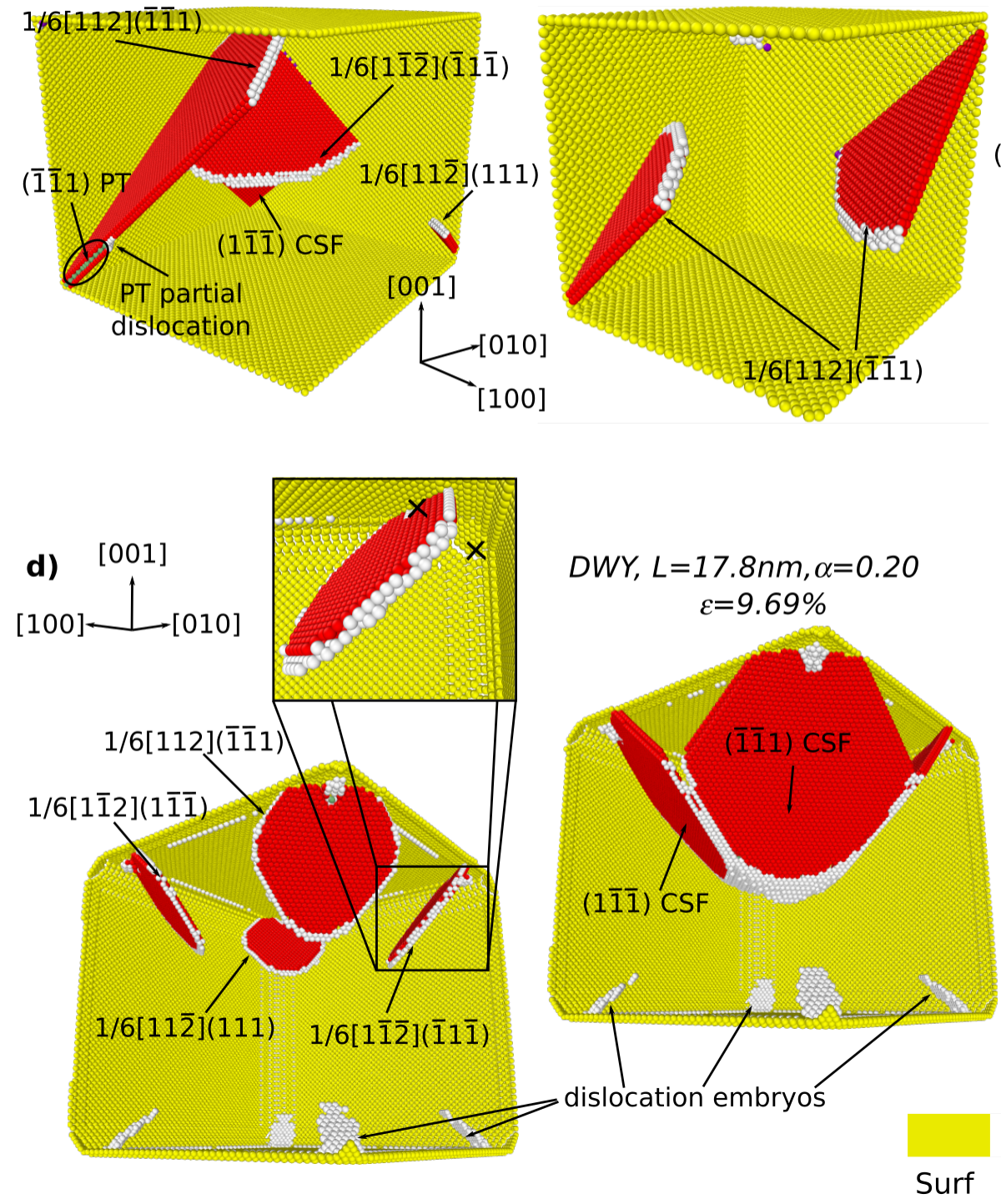

c) $\underset{\varepsilon=15.76 \%}{L=10.0 \mathrm{~nm}, \alpha=0.05}$

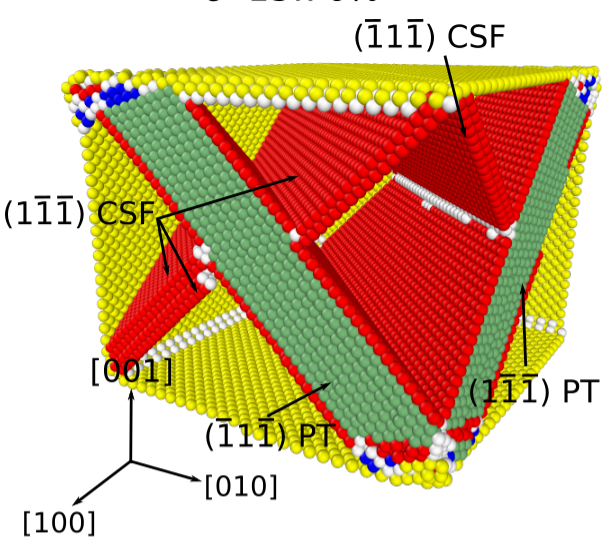

$$
\begin{gathered}
D W Y, L=17.8 n m, \alpha=0.20 \\
\varepsilon=10.11 \%
\end{gathered}
$$

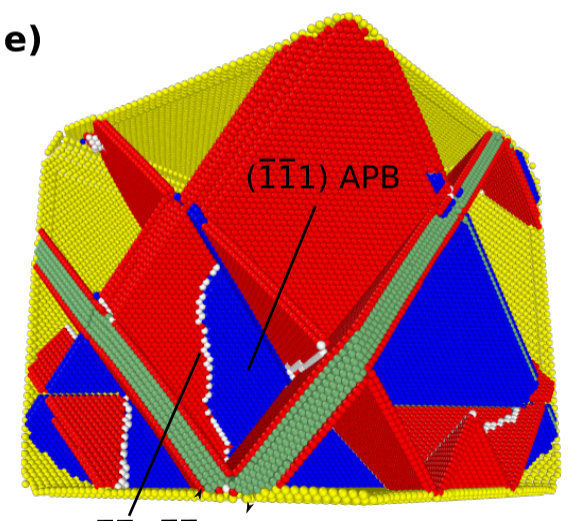

$1 / 6[1 \overline{2} \overline{1}](\overline{1} \overline{1} 1)$

Fig. 4 (color online) Surface dislocation nucleation in Ni $\mathrm{Al}_{\mathrm{A}} \mathrm{NPs}$. Atoms are coloured according to their respective crystalline environments (BAD analysis) as detailed in the colour box. Gold refers to surface atoms that are partially removed for better observations. White is for dislocation core atoms and sub-surface defective regions. Other colours rely on particular stacking environment. For a sake of clarity, atoms in perfect crystal environment are removed. a-b) Nucleation of partial dislocation from the corners of perfectly shaped NCs using DWY and AMB potentials, c) joint process of PT growth and surface dislocation nucleation at larger strain, $\boldsymbol{d})$ Two consecutive images of a typical dislocation nucleation event where multiple dislocations nucleate almost simultaneously. The inset shows the nucleation centre which is slightly shifted from the corner of the NP (black crosses refer to corner and dislocation nucleation site respectively). The second image shows the formation of a pyramidal dislocation structure. This structure is unstable and the NPs further deform by PT and partial dislocation nucleation as shown in 
e), where trailing partial dislocations and APBs are shown.

\subsection{High- $\alpha$ NPs deformation processes: transition to homogeneous dislocation nucleation}

Increasing $\alpha$ beyond a critical $\alpha$ ' value changes the elementary dislocation nucleation process where a heterogeneous-to-homogeneous dislocation nucleation transition takes place. For $\alpha>\alpha^{\prime}$, the first stress drop is characterized by the nucleation from the inside of the NP of a partial dislocation loop that quickly propagates through the entire sample (Fig. 5). One can note that there is no apparent singularity linked to the heterogeneous-to-homogeneous transition on the stress - strain curves (Figs. 2 and 3). This kind of homogeneous nucleation event happens shortly before the emission of subsequent dislocation from the corners

of the NPs and same slip systems (M 0.47) are involved in both nucleation regimes (Fig. 4 and 5). This original process occurs at $T=5$ and $300 \mathrm{~K}$ as well as in simulations using the AMB potential (see supplementary Figures S2, S3 and S4 for more details). After the first (homogeneous) nucleation event, a PT nucleates and grows, exactly as in the case of surface-dominated nucleation simulations.

Figure 5 shows several PT growing processes that involve the interaction of a pre-existing CSF with a freshly nucleated dislocation: (i) the homogeneous nucleation of a coplanar partial dislocation loop, one layer above (or below) the pre-existing CSF (Fig. 5b), (ii) the surface nucleation of a coplanar partial dislocation again one layer above (or beyond) the pre-existing CSF (Fig. 5c) and (iii) the homogeneous nucleation of a coplanar partial dislocation in the vicinity of intersecting CSFs (Fig. 5d). The growth of a PT results from the activation of a single process (e.g. the (111) PT observed in the $L=10 \mathrm{~nm}, \alpha=0.20 \mathrm{NP}$ as depicted on Fig. $5 \mathrm{~b}$ ) or from the simultaneous activation of several processes (e.g. a PT observed in the $L=15 \mathrm{~nm}, \alpha=0.20 \mathrm{NP}$, see Figure S5 in supplementary information).

Finally, the incipient plasticity of $\mathrm{Ni}_{3} \mathrm{Al}$ nanospheres $(\alpha=1)$ is characterized by the nucleation from the contact surface of a pyramidal dislocation structure comparable to the one already shown Figure 4d. 
This structure is similar to the "Pyramid Hillock dislocation structures" originally observed in FCC nanosphere compression tests $[20,50]$. This last result indicates that a backward homogeneous-to-heterogeneous nucleation transition occurs for larger $\alpha$ values $\left(\alpha^{\prime}<\alpha<1\right)$. While it will not be further detailed in this study, this secondary transition is observed for $\alpha=0.575 \pm 0.175$ and $\alpha=0.550 \pm 0.050$ for $L=5$ and $15 \mathrm{~nm}$ NPs respectively.

\section{Discussion}

\subsection{Stress definition and mechanical response}

The question of the stress definition is a recursive issue in nanomechanical MD simulations and smallscale experiments. In this context, the engineering compressive stress is frequently used in the literature due to both its ability to provide a clear description of the initial contact interaction between the sample and the indenter and its relative ease of use $[31,51,52]$. In this study, the counter-intuitive force softening versus stress strengthening behaviour (see Figure 2) is linked to the compressive stress definition itself as it arises from the accounted surface area. While in the simulation the yield force decreases with increasing $\alpha$, the yield stress increases due to a faster diminution of the contact area compared to the force decrease. Qualitatively, a similar behaviour is observed while using the true compressive stress definition that accounts for time-dependent contact surfaces. 
a)

$D W Y, L=17.8 \mathrm{~nm}, \alpha=0.25$

$\varepsilon=9.52 \%$

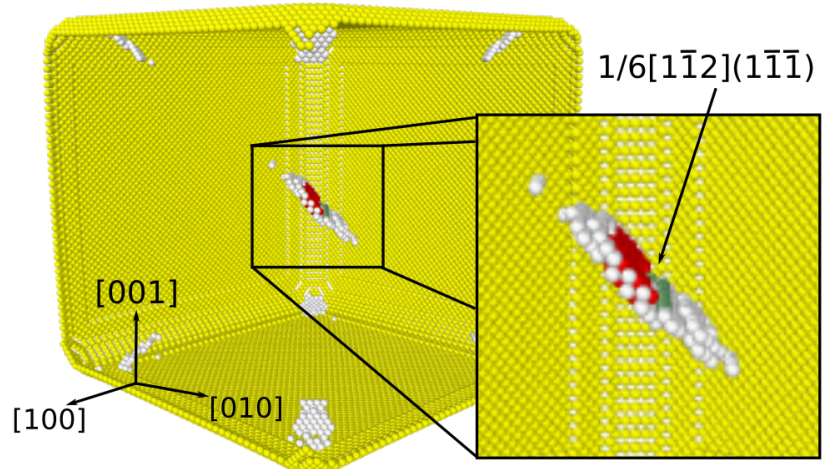

$D W Y, L=17.8 \mathrm{~nm}, \alpha=0.25$

c)

$\varepsilon=9.54 \%$

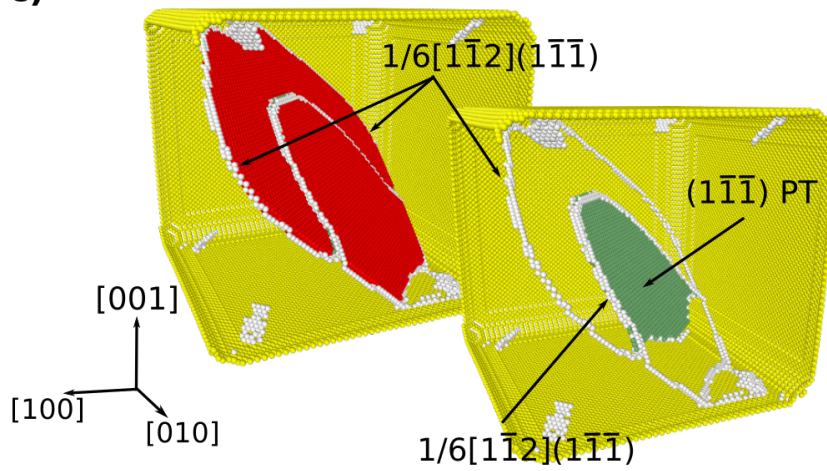

b)

$D W Y, L=10.0 \mathrm{~nm}, \alpha=0.20$ $\varepsilon=10.05 \%$

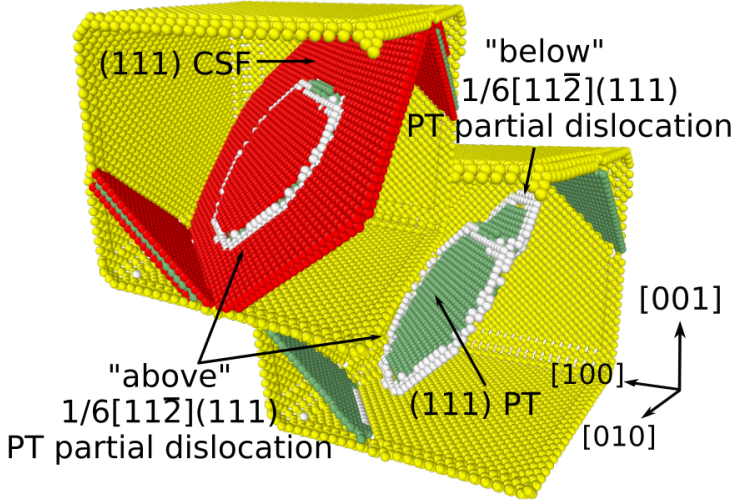

d)

$$
D W Y, L=17.8 \mathrm{~nm}, \alpha=0.50
$$
$\varepsilon=9.30 \%$

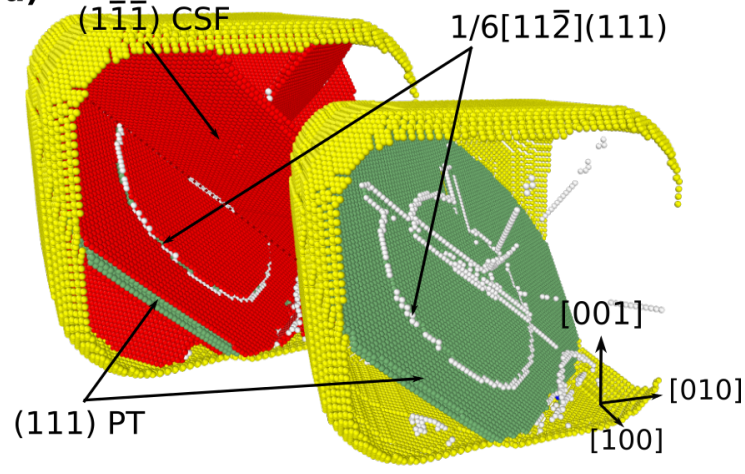

Fig. 5 (color online) Homogeneous dislocation nucleation and twin growth in blunt NPs. Atoms are coloured according to their crystalline environments (BAD analysis) as already described Fig. 4. Yellow, red, green and white atoms refer respectively to surface, CSF, PT and defective regions. To improve visibility, parts of the surface and perfect crystal atoms are removed. a) Homogeneous nucleation of a partial dislocation inside the NP, $\boldsymbol{b}$-c-d) Possible twin growth processes occurring after homogeneous dislocation nucleation. Two pictures with and without atoms in CSF environment (colored in red) are shown for a sake of clarity, b) keywords "above" and "below" refer to the positions of the two twinning dislocations relatively to the extended (111) CSF.

Nevertheless, one has to admit that measuring true contact area remains a relatively complicated task in the case of blunt NPs, especially in in situ experiments that only provide quasi-2D projections of the surface sample and generally do not allow for atomic resolution of the contact area during compression. In addition, high- $\alpha$ values simulations have shown some unexpected variations of the true contact area before yielding (during the elastic load) due to interplanar indentation phenomena i.e. when the top atomic layer sufficiently bends second and 
subsequent atomic layers as shown Figure 6. At this point, border atoms of subsequent atomic layers can reach the same $z$ value that first layer atoms leading to an increase of the contact surface that has not been measured in experiments up to now. Overall, these observations confirm that the engineering stress might be more appropriate to describe NPs compression tests regarding how doubtful could be the measure (or computation) of the true contact surface at the nanoscale.

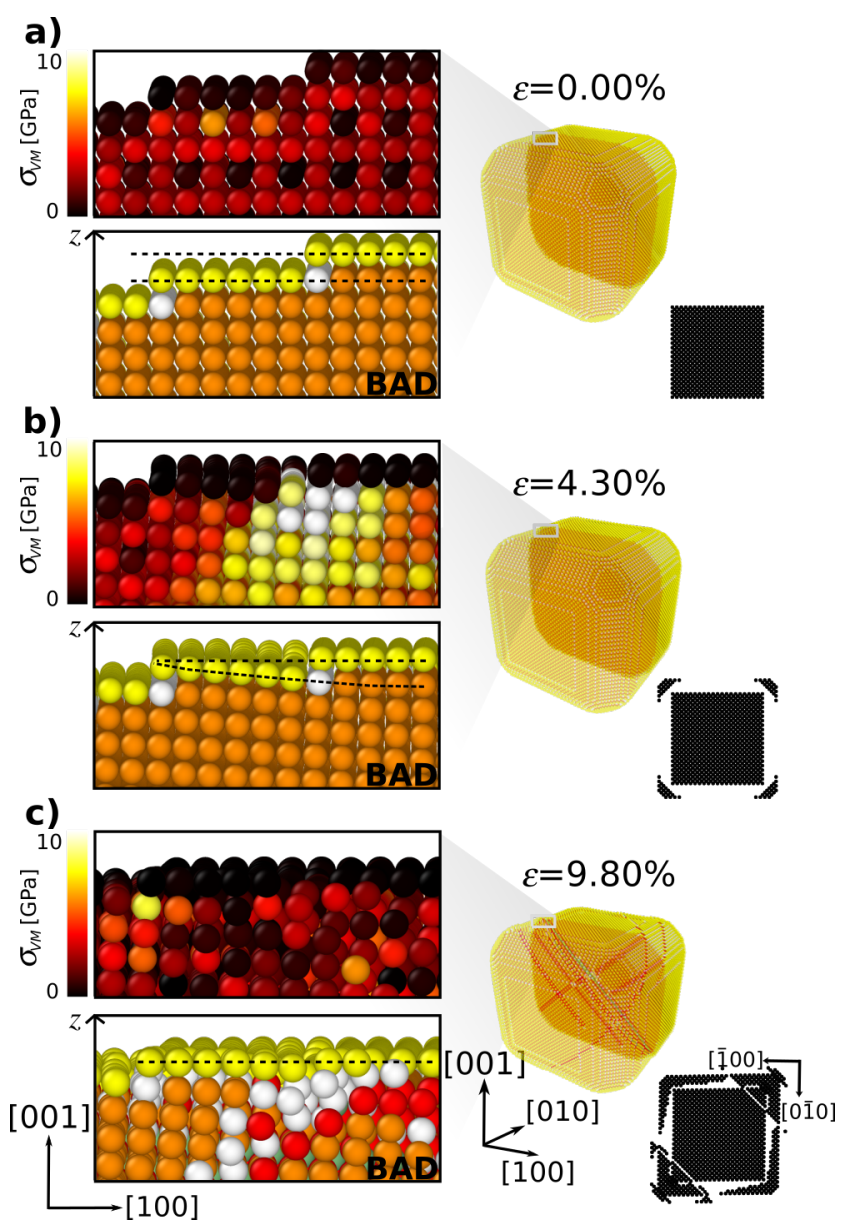

Fig. 6 (color online) Surface flattening and stress concentration during the compression of a $L=15 \mathrm{~nm} \mathrm{Ni} \mathrm{Al}_{\mathrm{A}} \mathrm{NP}$ $(\alpha=0.50)$ using the DWY parameterization. On the left, atoms are coloured according to both the local von Mises stress and the BAD analysis (already described Fig. 4). Black dotted lines are guides for the eyes that refer to the alignment of the first two top atomic layers. On the right, a perspective view of the sample as well as a projection along $z$ of top surface atoms $(0.5 \AA$ thick) are shown. a) Initial configuration where the two top atomic layers are perfectly oriented perpendicularly to [001], b) Configuration at $\varepsilon=4.3 \%$ (elastic regime) where the first atomic layer indents and partially merges the second layer inducing stress localization. Some atoms from the first two layers are at the same height $z$ what increases the contact surface (especially on the corners), c) Configuration at $\varepsilon=9.8 \%$ 
following the first dislocation nucleation events. The first two layers almost fully merges due to the plastic relaxation.

On the other hand, mid-height stress and strain definitions are also frequently used, especially in the fields of mechanical and civil engineering $[53,54]$. The mid-height area is particularly easy to measure (using e.g. digital image correlation) and could be particularly suited to the case of NP compression tests for which contact with the indenter is not fully constrained as previously discussed. In our simulations, the mid-height section area is computed on-the-fly using the same Hull algorithm that for the top surface calculation, applied here to a $2 \AA$ thick layer of atoms around NP mid-height and projected along the compression axis. Accordingly, the midheight stress behaves the opposite way when compared to the compressive stress i.e. the mid-height yield stress decreases with increasing $\alpha$ (see supplementary Figure S6). As for the compressive stress, the mid-height stress behaviour can be easily interpreted based on the competition between force decrease and section area variations with increasing $\alpha$. In this case, the mid-height area is less impacted by the NP smoothing procedure as only edge blunting is accounted, while both edges and corners blunting are taken into account in the $\alpha$-induced contact surface shrinking. Thus, mid-height area decreases slower than the indenter force (increasing $\alpha$ ) leading to a similar softening than the one observed on force - displacement curves (Fig. 2a).

Nevertheless, one can argue that while the mid-height stress can easily be defined in both MD simulations and in situ experiments, the classical compressive stress better describes close-to-surfaces regions where plastic deformation often initiates what makes it more appropriate. In any case and setting aside the force measured, the induced mechanical response is strongly linked to the choice and measurement of an effective section area that seems to be even more relevant for nano-objects for which a rigorous contact definition at the atomic scale remains hard to reach.

4.2 Stress concentration and dislocation nucleation 
As shown Figure 6, blunt edges and corners flatten during deformation and the top atomic layer bends underneath layers in a purely elastic manner inducing local stress concentration. Later during the compression, such configurations relax plastically when first dislocations nucleate. In order to better understand the link between mechanical response and dislocation nucleation processes, we investigate the internal shear stress distribution in both nucleation regimes (Figure 7).

In the case of perfectly shaped $\operatorname{NCs}(\alpha=0)$, the stress field is homogeneous within the whole sample except in corner regions, just before the first nucleation events. For samples with $0<\alpha<\alpha$, the local stress is particularly high close to the edges and corners that are in contact with the indenters just before the surface nucleation event. For $\alpha>\alpha$, stress fields intensify and overlap in the centre region of the sample leading to high shear stress concentration and homogeneous dislocation nucleation. These results are illustrated Figure 7 in the case of two $L=17.8 \mathrm{~nm}$ NPs $(\alpha=0$ and $\alpha=0.25$ ) for which the first dislocation nucleates from the same $\beta A$ $1 / 6[1 \overline{1} 2](1 \overline{1} \overline{1})$ slip system. While the stress field is almost homogeneous for the $\alpha=0$ NP leading to a surface nucleation event, it concentrates in the central part of the $\alpha=0.25\left(\alpha>\alpha^{\prime}\right)$ NP leading to homogeneous nucleation. Such stress concentration pattern has been observed using both DWY and the AMB interatomic potentials as well as in qualitative elastic FEM simulation tests. In the case depicted on Fig. 7c and d, the shear stress $\tau_{\beta A}$ in the centre region is about 4.5 GPa before nucleation which accounts for a macroscopic stress of about 9.6 GPa, below the (engineering compressive) yield strength $Y=13.3 \mathrm{GPa}$ what confirms the role of stress heterogeneities in homogeneous dislocation nucleation. As this phenomenon is induced by stress localization and geometry, we believe that a similar mechanical behaviour should be observed for other usual crystalline structures. 


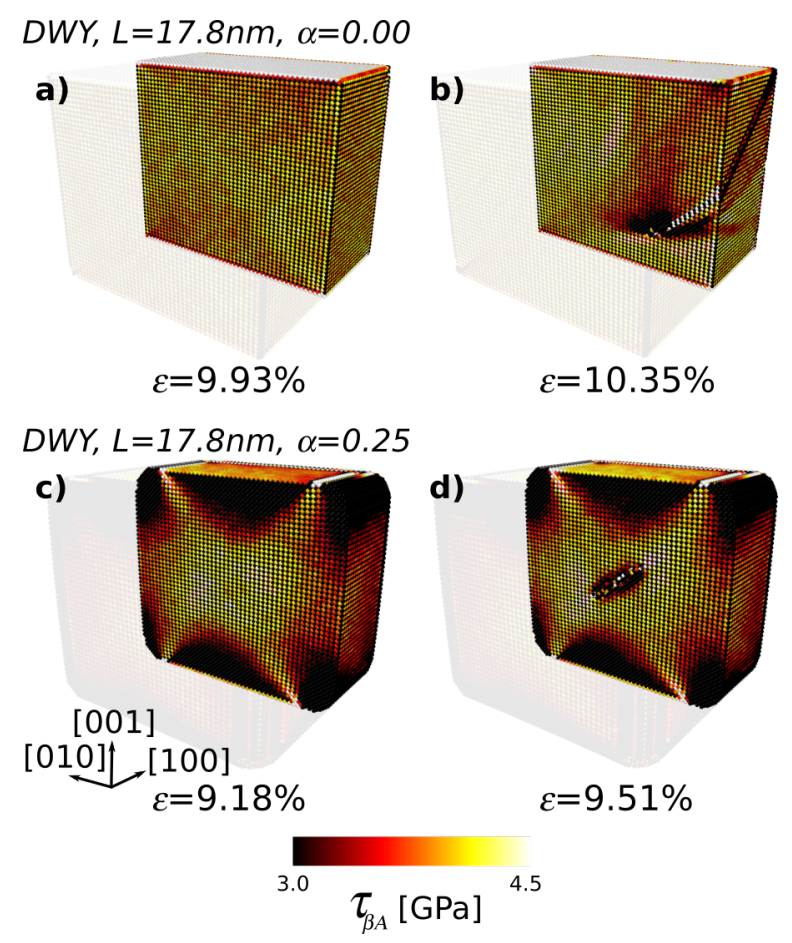

Fig. 7 (color online) Shear stress localization in two L=17.8 $\mathrm{nm} \mathrm{NPs} \mathrm{before} \mathrm{(left)} \mathrm{and} \mathrm{after} \mathrm{(right)} \mathrm{the} \mathrm{nucleation} \mathrm{of} \mathrm{a}$ dislocation in the $\beta A 1 / 6[1 \overline{1} 2](1 \overline{1} \overline{1})$ slip system. $\boldsymbol{a}$-b) Case of a perfectly shaped $N C(\alpha=0)$ in which the dislocation nucleates from a top corner. No stress heterogeneities are observed before dislocation nucleation except in the vicinity of the corners. A similar behaviour is observed in all NPs with $\alpha<\alpha^{\prime}, \boldsymbol{c}$-d) Case of a blunt NP with $\alpha=0.25$. In this case, the shear stress concentrates in the centre region of the sample, where the dislocation later nucleates. This process is specific to $\alpha>\alpha^{\prime} N P$ s.

While the central stress localization observed in MD might be overstated by the blunting process symmetries, we believe that more off-centre or sub-surface homogeneous nucleation events might happen in experimental samples as e.g. $\gamma^{\prime}$ precipitates [24,25], Au [19,55] and Ag [56] NPs as well as Si NCs [33] which are known to exhibit rounded corners and vertices. In this context, supplementary experimental investigations are required to verify the emergence of homogeneous nucleation events during the compression of blunt nano-objects.

4.3 A size-dependent shape-effect 


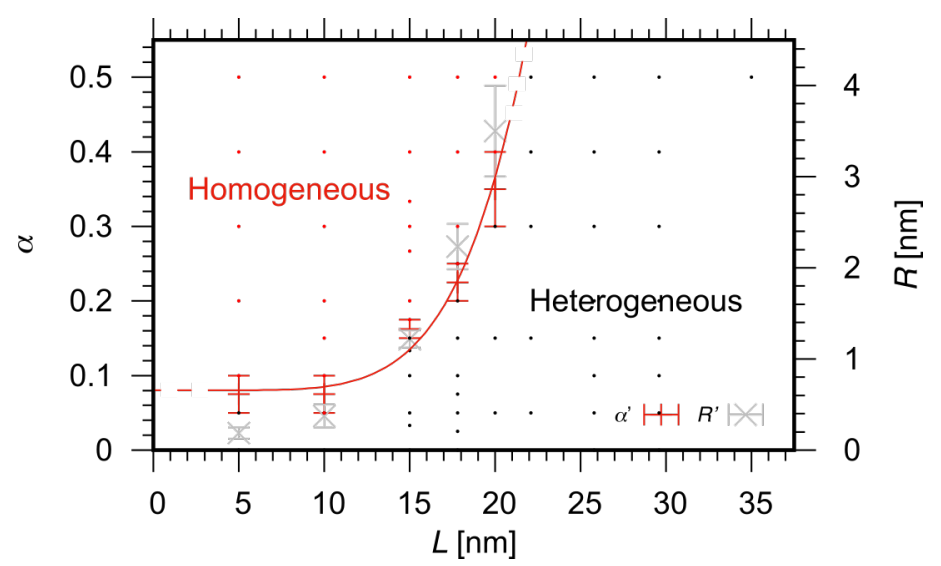

Fig. 8 (color online) Transition coefficients $\alpha^{\prime}$ (red) and $R^{\prime}$ (grey) function of the NPs size L. Black and red dots refer to the $(L, \alpha)$ conditions tested in the simulations leading to heterogeneous or homogeneous nucleation.

From our knowledge, the concept of homogeneous dislocation nucleation during nanoscale experiments is not discussed in the literature. One possible reason could be linked to the propagation rate at which a (freshly nucleated) dislocation would reach the sample surface after a homogeneous nucleation event leaving the sample in the same state than surface nucleation events. In this study, this case is illustrated in Figures $4 \mathrm{~b}$ and $5 \mathrm{~b}$ in which similar extended stacking faults that originate respectively from heterogeneous and homogeneous nucleation processes are shown. This feature makes the nucleation character authentication (heterogeneous or homogeneous) difficult in both MD simulations and in situ experiments. Nevertheless, there is at least one other well-known example where homogeneous nucleation happens: the nanoindentation case [57,58]. The comparison between our simulations and nanoindentation is particularly relevant as nanoindentation involves a blunt shaped tip indenting a flat sample what is somehow the reverse configuration when compared to our simulation setup i.e. a blunt shaped NP indented by a flat indenter. In Miller and Rodney [57], the authors emphasize that sub-surface homogeneous nucleation should be restricted to a certain extent of the indenter size due to an increase of the critical dislocation loop radius. To confront this argument and thus test the hypothetical size-dependency of the heterogeneous-to-homogeneous dislocation nucleation transition, supplementary simulations have been performed for $L=20.0,22.1,26.5$ and $35 \mathrm{~nm}$ (Figure 8). The size versus 
shape analysis depicted on Figure 8 shows that homogeneous nucleation occurs in NPs with sizes ranging from 5 to $20 \mathrm{~nm}$ with $\alpha^{\prime}$ increasing from $0.075 \pm 0.025$ to $0.35 \pm 0.05$ while larger NPs only show heterogeneous dislocation nucleation. These results confirm that the homogeneous dislocation nucleation process which is originally induced by the relative indenter/sample shape in NPs also depends on size, as in the case of nanoindentation. In the following, we propose a simple model to justify $\alpha$ ' shape vs. size dependency.

Assuming that homogeneous dislocation nucleation relies on the ease of first atomic layers to indent each other

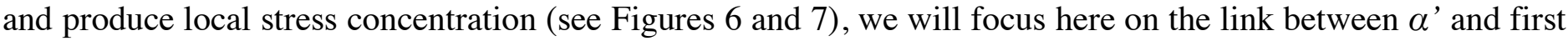
layers characteristic lengths. In a simplified 2D approach (see supplementary Figure S7), the diminution of the atomic layers characteristic length induced by the blunting process can be described by equation (1):

$L_{n}=L_{1}+2 x_{n}$ with $1 \leq n \leq n_{\text {lim }}$

Where $L_{n}$ is the characteristic length of the $n^{\text {n }}$ layer of atoms (starting from the contact surface) and $x_{n}$ is a length increment. $n_{\text {lim }}$ relies on the last layers impacted by the blunting process. While $L_{1}=L-2 R$, subsequent $L_{n}$ lengths are easily deduced from a combination of $L_{l}$ and $d_{\infty l}$, the (001) interplanar distance and $x_{n}$ can be expressed as follows:

$x_{n}=(n-1) d_{001} \sqrt{\frac{2 R}{(n-1) d_{001}}-1}$

Focusing on the first two atomic layers and assuming that the interlayer indentation ease and thus, the stress concentration, scale with the ratio between characteristic lengths $L_{2} / L_{l}$ (no interlayer indentation for the NC case $L_{2} / L_{l}=1$ but only for blunt NPs where $\left.L_{2} / L_{l}>1\right), \alpha^{\prime}$ should scale with the inverse ratio $L_{l} / L_{2}$ :

$\alpha^{\prime} \propto \frac{L_{1}}{L_{2}}=\frac{1}{1+\frac{d_{001}}{\frac{L}{2}-R} \sqrt{\frac{2 R}{d_{001}}-1}}$

Equation (3) shows that the $L_{l} / L_{2}$ ratio (and thus $\alpha^{\prime}$ ) increases non-linearly when $L$ increases and becomes closer to the ideal NC case $\left(L_{l} / L_{2}=1\right)$ typical of the heterogeneous regime of dislocation nucleation in spite of any $R$ variations. 
This simple model correctly reproduces the trend of the results presented Figure 8 assuming a low- $L$ cut-off and justifies the size dependency of the homogeneous-to-heterogeneous dislocation nucleation transition.

\section{Conclusion}

In summary, MD simulations based on semi-empiric interatomic potentials have been used to investigate how blunt shapes may influence both the mechanical strength and the incipient plasticity processes of nanocrystals by rounding edges and vertices of originally cubic-shaped NCs. While the lack of size-effect on the strength of $\mathrm{L}_{2} \mathrm{Ni}_{3} \mathrm{Al} \mathrm{NCs}$ is confirmed, a strong shape-effect is shown i.e. smoothing corners and edges of

originally cubic particles leads to strengthening. This property might be one of the possible reason for yield strength discrepancies as measured in SEM and in situ TEM mechanical tests (see e.g. ref.[25] for $\mathrm{Ni}_{3} \mathrm{Al} \mathrm{NPs}$ data). Furthermore, here we have shown that homogeneous dislocation nucleation could also happen in blunt NPs in a like manner to nanoindentation. We believe that a similar mechanism could also apply in other nanostructures like nanowires and pillars depending on their surface state and supplementary experimental investigations would be required to examine possible homogeneous nucleation events. This study emphasizes how much the design of virtual samples is crucial to model nano-objects mechanical properties and provides new insights into incipient plasticity features at the nanoscale.

\section{Acknowledgments}

The authors thank Dr. E. Maras, Dr. S. Dezecot and Dr. A. Goryaeva for useful discussions and comments. This work was granted access to the HPC resources (P2CHPD) of the Fédération Lyonnaise de Modélisation et Sciences Numériques (FLMSN), partner of the EQUIPEX EQUIP@MESO.

\section{References}

[1] H.G. Craighead, Nanoelectromechanical systems, Science. 290 (2000) 1532-1535. 
doi:10.1126/science.290.5496.1532.

[2] O.Y. Loh, H.D. Espinosa, Nanoelectromechanical contact switches, Nat. Nanotechnol. 7 (2012) 283295. doi:10.1038/nnano.2012.40.

[3] P. Alivisatos, The use of nanocrystals in biological detection, Nat Biotechnol. 22 (2003) 47-52. doi: $10.1038 /$ nbt927.

[4] P.D. Howes, R. Chandrawati, M.M. Stevens, Bionanotechnology. Colloidal nanoparticles as advanced biological sensors, Science. 346 (2014) 1247390-1247390. doi:10.1126/science.1247390.

[5] Y. Liu, X. Sun, S. Wang, M. Xie, A. Chen, R. Long, Preparation of nanoparticles embedded microcapsules (NEMs) and their application in drug release, Mater. Lett. 75 (2012) 48-50. doi:10.1016/j.matlet.2012.01.137.

[6] X. Lin, T. Bai, Y.Y.Zuo, N. Gu, Promote potential applications of nanoparticles as respiratory drug carrier: insights from molecular dynamics simulations, Nanoscale. 6 (2014) 2759-9. doi:10.1039/c3nr04163h.

[7] R. Tenne, L. Rapoport, Y. Bilik, Y. Feldman, M. Homyonfer, S.R. Cohen, Hollow Nanoparticles of $\mathrm{WS}_{2}$ as Potential Solid-State Lubricants, Nature. 387 (1997) 791-793. doi:10.1038/42910.

[8] A. Hernández Battez, R. González, J.L. Viesca, J.E. Fernández, J.M. Díaz Fernández, A. Machado, et al., $\mathrm{CuO}, \mathrm{ZrO}_{2}$ and $\mathrm{ZnO}$ nanoparticles as antiwear additive in oil lubricants, Wear. 265 (2008) 422428. doi:10.1016/j.wear.2007.11.013.

[9] H. Liang, M. Upmanyu, H. Huang, Size-dependent elasticity of nanowires: nonlinear effects, Phys. Rev. B. 71 (2005) 241403. doi:10.1103/PhysRevB.71.241403.

[10] M.T. McDowell, A.M. Leach, K. Gaill, On The Elastic Modulus of Metallic Nanowires, Nano Lett. 8 (2008) 3613-3618. doi:10.1021/n1801526c.

[11] O. Kraft, P.A. Gruber, R. Mönig, Plasticity in confined dimensions, Annu. Rev. Mater. Res. 40 (2010) 293-317. doi:10.1146/annurev-matsci-082908-145409.

[12] J. Greer, J. De Hosson, Plasticity in small-sized metallic systems: Intrinsic versus extrinsic size effect, Prog. Mater. Sci. 56 (2011) 654-724. doi:10.1016/j.pmatsci.2011.01.005.

[13] S.H. Oh, M. Legros, D. Kiener, G. Dehm, In situ observation of dislocation nucleation and escape in a submicrometre aluminium single crystal, Nat. Mater. 8 (2009) 95-100. doi:10.1038/nmat2370.

[14] Q. Yu, M. Legros, A.M. Minor, In situ TEM nanomechanics, MRS Bulletin. 40 (2015) 62-70. doi:10.1557/mrs.2014.306.

[15] C.R. Weinberger, W. Cai, Surface-controlled dislocation multiplication in metal micropillars, Proc. Natl. Acad. Sci. U.S.a. 105 (2008) 14304-14307. doi:10.1073/pnas.0806118105.

[16] C. Deng, F. Sansoz, Near-Ideal Strength in Gold Nanowires Achieved through Microstructural Design, ACS Nano. 3 (2009) 3001-3008. doi:10.1021/nn900668p.

[17] J. Guénolé, J. Godet, S. Brochard, Deformation of silicon nanowires studied by molecular dynamics simulations, Model. Sim. Mat. Sci. Eng. 19 (2011) 074003-126. doi:10.1088/0965-0393/19/7/074003.

[18] S. Narayanan, G. Cheng, Z. Zeng, Y. Zhu, T. Zhu, Strain Hardening and Size Effect in Five-fold Twinned Ag Nanowires, Nano Lett. (2015) 150515102410001-8. doi:10.1021/acs.nanolett.5b01015.

[19] D. Mordehai, S.-W. Lee, B. Backes, D.J. Srolovitz, W.D. Nix, E. Rabkin, Size effect in compression of single-crystal gold microparticles, Acta Mater. 59 (2011) 5202-5215. doi:10.1016/j.actamat.2011.04.057.

[20] J.-J. Bian, G.-F. Wang, Atomistic Deformation Mechanisms in Copper Nanoparticles, Jnl of Comp \& Theo Nano. 10 (2013) 2299-2303. doi:10.1166/jctn.2013.3201.

[21] C.R. Weinberger, W. Cai, Plasticity of metal nanowires, J. Mater. Chem. 22 (2012) 3277. doi:10.1039/c2jm13682a.

[22] J. Amodeo, E. Bitzek, C. Begau, Atomistic Simulations of Compression Tests on $\mathrm{Ni}_{3} \mathrm{Al}$ Nanocubes, Mat. Res. Lett. 2 (2014) 140-145. doi:10.1080/21663831.2013.878884. 
[23] K. Shreiber, D. Mordehai, Dislocation-nucleation-controlled deformation of $\mathrm{Ni}_{3} \mathrm{Al}$ nanocubes in molecular dynamics simulations, Model. Sim. Mat. Sci. Eng. (2015) 1-17. doi:10.1088/09650393/23/8/085004.

[24] J. Schloesser, J. Roesler, D. Mukherji, Deformation behaviour of freestanding single-crystalline $\mathrm{Ni}_{3} \mathrm{Al}-$ based nanoparticles, Int. J. Mater. Res. 102 (2011) 532-537. doi:10.3139/146.110504.

[25] R. Maßß, L. Meza, B. Gan, S. Tin, J.R. Greer, Ultrahigh strength of dislocation-free $\mathrm{Ni}_{3} \mathrm{Al}$ nanocubes, Small. 8 (2012) 1869-1875. doi:10.1002/smll.201102603.

[26] L. Kovarik, R.R. Unocic, J. Li, P. Sarosi, C. Shen, Y. Wang, et al., Microtwinning and other shearing mechanisms at intermediate temperatures in Ni-based superalloys, Prog. Mater. Sci. 54 (2009) 839873. doi:10.1016/j.pmatsci.2009.03.010.

[27] H.-X. Xie, L. Bo, T. Yu, Atomistic simulation of microtwinning at the crack tip in $\mathrm{L}_{2} \mathrm{Ni}_{3} \mathrm{Al}$, Philos. Mag. A. 92 (2012) 1542-1553. doi:10.1080/14786435.2011.652690.

[28] C.R. Weinberger, A.T. Jennings, K. Kang, J.R. Greer, Atomistic simulations and continuum modeling of dislocation nucleation and strength in gold nanowires, J. Mech. Phys. Solids. 60 (2012) 84-103. doi:10.1016/j.jmps.2011.09.010.

[29] P. Valentini, W. Gerberich, T. Dumitrica, Phase-Transition Plasticity Response in Uniaxially Compressed Silicon Nanospheres, Phys. Rev. Lett. 99 (2007) 175701.

doi:10.1103/PhysRevLett.99.175701.

[30] T.W. Stone, M.F. Horstemeyer, Length scale effects of friction in particle compaction using atomistic simulations and a friction scaling model, J Nanopart Res. 14 (2012) 1121-13. doi:10.1007/s11051-0121121-0.

[31] D. Mordehai, S.-W. Lee, B. Backes, D.J. Srolovitz, W.D. Nix, E. Rabkin, Size effect in compression of single-crystal gold microparticles, Acta Mater. 59 (2011) 5202-5215.

doi:10.1016/j.actamat.2011.04.057.

[32] D.D. Stauffer, A. Beaber, A. Wagner, O. Ugurlu, J. Nowak, K.A. Mkhoyan, et al., Strain-hardening in submicron silicon pillars and spheres, Acta Mater. 60 (2012) 2471-2478.

doi:10.1016/j.actamat.2011.10.045.

[33] A.J. Wagner, E.D. Hintsala, P. Kumar, W.W. Gerberich, K.A. Mkhoyan, Mechanisms of plasticity in near-theoretical strength sub-100nm Si nanocubes, Acta Mater. 100 (2015) 256-265.

doi:10.1016/j.actamat.2015.08.029.

[34] Y.M. Wang, Defective twin boundaries in nanotwinned metals, Nat. Mater. 12 (2013) 697-702. doi:10.1038/nmat3646.

[35] J.R. Greer, Nanotwinned metals: It's all about imperfections, Nat. Mater. 12 (2013) 689-690. doi:10.1038/nmat3721.

[36] D. Pope, S. Ezz, Mechanical properties of $\mathrm{Ni}_{3} \mathrm{AI}$ and nickel-base alloys with high volume fraction of $\gamma^{\prime}$, Int. Met. Rev. 29 (1984) 136-167. doi:10.1179/imtr.1984.29.1.136.

[37] R.C. Reed, The superalloys: fundamentals and applications, Cambridge University Press, New York, 2008. doi:10.1017/CBO9780511541285.

[38] S. Plimpton, Fast Parallel Algorithms for Short-Range Molecular-Dynamics, J. Comput. Phys. 117 (1995) 1-19. doi:10.1006/jcph.1995.1039.

[39] J.P. Du, C.Y. Wang, T. Yu, Construction and application of multi-element EAM potential (Ni-Al-Re) in $\gamma / \gamma^{\prime}$ Ni-based single crystal superalloys, Model. Sim. Mat. Sci. Eng. 21 (2013). doi:10.1088/09650393/21/1/015007.

[40] J.E. Angelo, N.R. Moody, M.I. Baskes, Trapping of Hydrogen to Lattice-Defects in Nickel, Model. Sim. Mat. Sci. Eng. 3 (1995) 289-307. doi:10.1088/0965-0393/3/3/001.

[41] M.I. Baskes, X.W. Sha, J.E. Angelo, N.R. Moody, Trapping of hydrogen to lattice defects in nickel, Model. Sim. Mat. Sci. Eng. 5 (1997) 651-652. doi:10.1088/0965-0393/5/6/007. 
[42] Y. Mishin, Atomistic modeling of the $\gamma$ and $\gamma^{\prime}$-phases of the Ni-Al system, Acta Mater. 52 (2004) 1451-1467. doi:10.1016/j.actamat.2003.11.026.

[43] G.P. Purja Pun, Y. Mishin, Development of an interatomic potential for the Ni-Al system, Philos. Mag. A. 89 (2009) 3245-3267. doi:10.1080/14786430903258184.

[44] I. Issa, J. Amodeo, J. Réthoré, L. Joly-Pottuz, C. Esnouf, J. Morthomas, et al., In situ investigation of $\mathrm{MgO}$ nanocube deformation at room temperature, Acta Mater. 86 (2015) 295-304. doi:10.1016/j.actamat.2014.12.001.

[45] C.B. Barber, D.P. Dobkin, H. Huhdanpaa, The Quickhull algorithm for convex hulls, ACM Trans. on Math. Soft. 22 (1996) 469-483. doi:10.1145/235815.235821.

[46] C.S. Hartley, Y. Mishin, Characterization and visualization of the lattice misfit associated with dislocation cores, Acta Mater. 53 (2005) 1313-1321. doi:10.1016/j.actamat.2004.11.027.

[47] C. Begau, A. Hartmaier, E.P. George, G.M. Pharr, Atomistic processes of dislocation generation and plastic deformation during nanoindentation, Acta Mater. 59 (2011) 934-942.

doi:10.1016/j.actamat.2010.10.016.

[48] C. Begau, AtomViewer, Free Download at Http://Homepage.Ruhr-Uni-Bochum.De/Christoph.Begau/. (2014).

[49] A. Stukowski, Visualization and analysis of atomistic simulation data with OVITO-the Open Visualization Tool, Model. Sim. Mat. Sci. Eng. 18 (2010) 7pp. doi:10.1088/0965-0393/18/1/015012.

[50] S. Bel Haj Salah, C. Gerard, L. Pizzagalli, Influence of surface atomic structure on the mechanical response of aluminum nanospheres under compression, Comp. Mat. Sci. 129 (2017) 273-278. doi:10.1016/j.commatsci.2016.12.033.

[51] M.D. Uchic, D.M. Dimiduk, J.N. Florando, W.D. Nix, Sample Dimensions Influence Strength and Crystal Plasticity, Science. 305 (2004) 986-989. doi:10.1126/science.1098993.

[52] P.A. Shade, R. Wheeler, Y.S. Choi, M.D. Uchic, D.M. Dimiduk, H.L. Fraser, A combined experimental and simulation study to examine lateral constraint effects on microcompression of singleslip oriented single crystals, Acta Mater. 57 (2009) 4580-4587. doi:10.1016/j.actamat.2009.06.029.

[53] I. Georgieva, L. Schueremans, L. Pyl, Composed columns from cold-formed steel Z-profiles: Experiments and code-based predictions of the overall compression capacity, Eng. Struct. 37 (2012) 125-134. doi:10.1016/j.engstruct.2011.12.017.

[54] Q.-L. Wang, Z.Zhao, Y.-B. Shao, Q.-L. Li, Static behavior of axially compressed square concrete filled CFRP-steel tubular (S-CF-CFRP-ST) columns with moderate slenderness, Thin Walled Struct. 110 (2017) 106-122. doi:10.1016/j.tws.2016.10.019.

[55] M. Ramos, L. Ortiz-Jordan, A. Hurtado-Macias, S. Flores, J. Elizalde-Galindo, C. Rocha, et al., Hardness and Elastic Modulus on Six-Fold Symmetry Gold Nanoparticles, Materials. 6 (2013) 198205. doi:10.3390/ma6010198.

[56] C.E. Carlton, P.J. Ferreira, In situ TEM nanoindentation of nanoparticles, Micron. 43 (2012) 11341139. doi:10.1016/j.micron.2012.03.002.

[57] R. Miller, D. Rodney, On the nonlocal nature of dislocation nucleation during nanoindentation, J. Mech. Phys. Solids. 56 (2008) 1203-1223. doi:10.1016/j.jmps.2007.10.005.

[58] K. Xiong, H. Lu, J. Gu, Atomistic simulations of the nanoindentation-induced incipient plasticity in $\mathrm{Ni}_{3} \mathrm{Al}$ crystal, Comp. Mat. Sci. 115 (2016) 214-226. doi:10.1016/j.commatsci.2015.12.045. 